\title{
Categorical Models for a Semantically Linear $\lambda$-calculus*
}

\author{
Marco Gaboardi \\ Dipartimento di Informatica \\ Università degli Studi di Torino \\ gaboardi@di.unito.it
}

\author{
Mauro Piccolo \\ Dipartimento di Informatica \\ Università degli Studi di Torino \\ Preuves, Programmes et Systèmes \\ Université de Paris VII \\ piccolo@di.unito.it
}

\begin{abstract}
This paper is about a categorical approach to model a very simple Semantically Linear $\lambda$ calculus, named $\mathcal{S} \ell \lambda$-calculus. This is a core calculus underlying the programming language $\mathcal{S} \ell$ PCF. In particular, in this work, we introduce the notion of $\mathcal{S} \ell \lambda$-Category, which is able to describe a very large class of sound models of $\mathcal{S} \ell \lambda$-calculus. $\mathcal{S} \ell \lambda$-Category extends in the natural way Benton, Bierman, Hyland and de Paiva's Linear Category, in order to soundly interpret all the constructs of $\mathcal{S} \ell \lambda$-calculus. This category is general enough to catch interesting models in Scott Domains and Coherence Spaces.
\end{abstract}

\section{Introduction}

$\mathcal{S} \ell \lambda$-calculus - acronym for Semantically linear $\lambda$-calculus - is a simple term calculus based on $\lambda$-calculus. More specifically, $\mathcal{S} \ell \lambda$-calculus extends and refines simply typed $\lambda$-calculus by imposing a linearity discipline on the usage of certain kinds of variables, as well as by adding some programming features to the calculus - like numerals, conditional and fix point operators - to make the calculus expressive enough to program all first-order computable functions.

Semantically Linear $\lambda$-calculus was already introduced in [10] (with an additional operator called which?, that is not present here) as the term rewriting system on which the programming language $\mathcal{S} \ell$ PCF is based [3, 10]. S\&PCF is based on a syntactical restriction of PCF conceived in order to program only linear functions between Coherence Spaces. In particular, in [10] we define a concrete model of $\mathcal{S} \ell$ PCF (and consequently of $\mathcal{S} \ell \lambda$-calculus) in the category Coh of Coherence Spaces and Linear Functions, for which we prove a full abstraction result.

The aim of this paper is to give an abstract description of models of $\mathcal{S} \ell \lambda$-calculus. This in order to highlight the properties that a mathematical structure must satisfy to model, by means of its equational theory, the operational theory induced by the reduction rules of the calculus. We give this abstract description in terms of category theory and we show that the obtained notion can be used to build concrete models in different mathematical structures.

We recall that the category Coh, as well as many other categories, is a well known concrete instance of Benton, Bierman, Hyland and de Paiva's Linear Categories, introduced in [1] to provide an abstract description of models of Intuitionistic Linear Logic. All these categories are symmetric monoidal closed and they are equipped with a symmetric monoidal comonad ! used to interpret the exponential modality and satisfying certain properties [1]. The idea is to impose enough conditions on the comonad in order to make its induced Kleisli category a Cartesian Closed Category with exponential object $A \Rightarrow B=! A \multimap B$. The original construction

*Work partially supported by MIUR-PRIN'07 CONCERTO Project.

M. Florido and I. Mackie (Eds.): First International Workshop on Linearity (LINEARITY 2009)

EPTCS 22, 2010, pp. 1-13, doi 10.4204/EPTCS.22.1 (c) Marco Gaboardi and Mauro Piccolo This work is licensed under the Creative Commons Attribution License. 
does not require this, but it would actually be the case, if the monoidal closed category is also cartesian.

In this paper, we introduce the notion of $\mathcal{S} \ell \lambda$-Category which extends in the natural way the definition of Linear Category, in order to be able to interpret all programming constructs of $\mathcal{S} \ell \lambda$-calculus.

We ask that this category admits a morphism acting like a "conditional" and a morphism acting like a "fix-point operator". The latter turns out to be the expected decomposition of a fix-point morphism in a Cartesian Closed Category. Furthermore, to interpret ground values, we require the existence of a distinguished object $N$ with the usual zero and successor and predecessor morphisms satisfying the expected equations. However, since variables of ground type can be freely duplicated and erased, we need to ask that all numeral morphisms behaves properly with respect to the comonad !. For this purpose we ask the existence of !-coalgebra $p: N \rightarrow ! N$ which is also comonoidal and moreover we ask that all numeral morphisms are both coalgebraic and comonoidal.

The notion of natural number object in a symmetric monoidal closed category is not new and it was introduced by Paré and Román in [11]. Based on this definition Mackie, Román and Abramsky introduced an internal language for autonomous categories with natural number objects in [6]. The main similarity between the definitions of natural number object given in [11, 6] and our definition is the requirement of comonoidality of the natural number object; moreover their definition does not take into consideration the relationship between the natural number object and the exponential comonad !; in fact there, only a strictly linear language without exponential was analyzed. More details on this matter can be found in [12].

We prove that the proposed categorical model of $\mathcal{S} \ell \lambda$-calculus enjoys soundness with respect to the smallest equivalence containing $\mathcal{S} \ell \lambda$-reduction. This Soundness Theorem relies on three distinct substitution lemmas corresponding to the three kind of substitution in the calculus.

Moreover, this abstract definition of model for $\mathcal{S} \ell \lambda$-calculus allows us to analyze in a modular way many different concrete examples. In particular, we build a non-trivial model of $\mathcal{S} \ell \lambda$-calculus in the category StrictBcdom of Scott Domains and strict continuous functions. We also study models of $\mathcal{S} \ell \lambda$-calculus in the category Coh of Coherence Spaces and linear stable functions and in the category StrictBcdom of Scott Domains and linear functions. More specifically, this implies that the model we defined in [10] is equivalent to a particular instance of $\mathcal{S} \ell \lambda$-Category, in the category Coh.

Finally, we address the completeness of the $\mathcal{S} \ell \lambda$-calculus with respect to $\mathcal{S} \ell \lambda$-category. We show that the completeness with respect to the standard interpretation fails. So we discuss some ways to recover it.

We conclude by giving some possible future directions.

\section{Semantically Linear $\lambda$-calculus}

$\mathcal{S} \ell \lambda$-calculus is a term rewriting system very close to $\lambda$-calculus, on which the programming language $\mathcal{S} \ell$ PCF is based [3, 10]. Truth-values of $\mathcal{S} \ell \lambda$-calculus are encoded as integers (zero encodes "true" while any other numeral stands for "false"). The set of $\mathcal{S} \ell \lambda$-types is defined as, $\sigma, \tau::=\iota \mid(\sigma \multimap \tau)$ where $\iota$ is the only atomic type (i.e. natural numbers), $\multimap$ is the only type constructor and $\sigma, \tau, \ldots$ are meta-variables ranging over types. Let $\operatorname{Var}^{\sigma}, \operatorname{SVar}^{\sigma}$ be enumerable disjoint sets of variables of type $\sigma$. The set of ground variables is $\mathrm{Var}^{l}$, the set of higher-order 


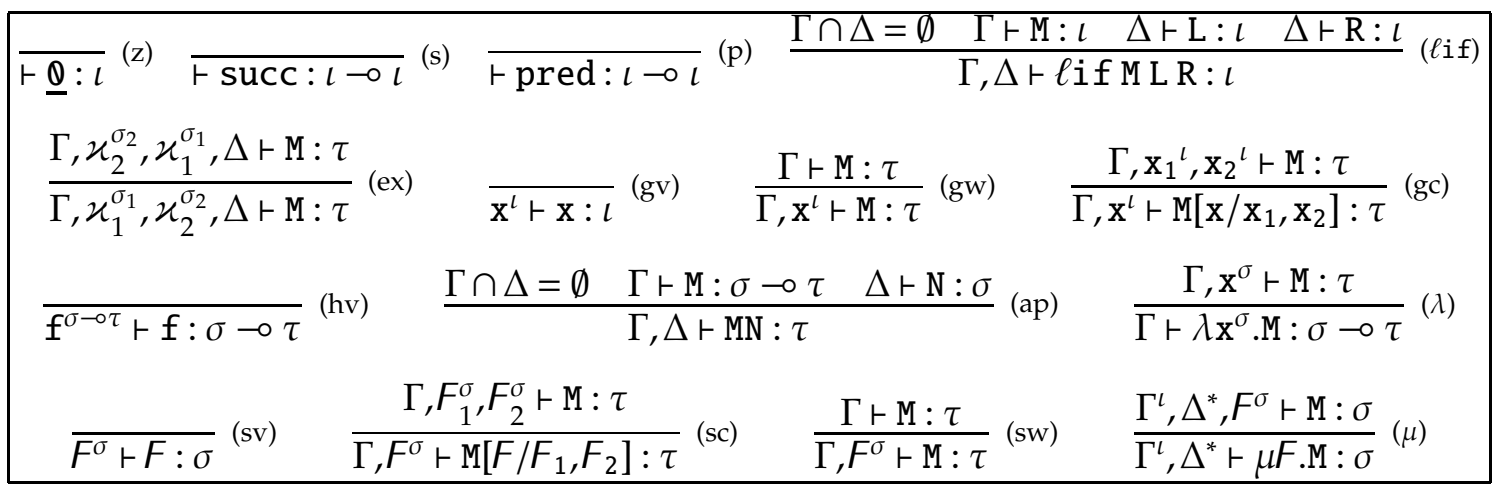

Table 1: Type assignment system for $\mathcal{S} \ell \lambda$-calculus

variables is HVar $=\bigcup_{\sigma, \tau} \operatorname{Var}^{\sigma-\sigma \tau}$, the set of stable variables is SVar $=\bigcup_{\sigma} \operatorname{SVar}^{\sigma}$ and the whole set of variables is $\operatorname{Var}=\operatorname{Var}^{l} \cup$ HVar $\cup$ SVar. Letters $x^{\sigma}$ range over variables in $\operatorname{Var}^{\sigma}$, letters $\mathrm{y}^{\iota}, \mathrm{z}^{\iota}, \ldots$ range over variables in $\operatorname{Var}^{\iota}$, letters $\mathrm{f}^{\sigma-\circ \tau}, \mathrm{g}^{\sigma-0 \tau}, \ldots$ range over variables in HVar, while $F^{\sigma}, F_{1}{ }^{\sigma}, F_{2}{ }^{\sigma}, \ldots$ range over stable variables, namely variables in SVar ${ }^{\sigma}$. Last, $u$ will denote any kind of variables. Latin letters $\mathrm{M}, \mathrm{N}, \mathrm{L}, \ldots$ range over terms.

A basis $\Gamma$ is a finite list of variables in Var. We denote with $\Gamma^{*}\left(\operatorname{resp} \Gamma^{\iota}\right)$ a basis $\Gamma$ containing variables in SVar (resp. in $\left.\operatorname{Var}^{l}\right)$. We will denote with $\Gamma, \Delta$ the concatenation of two basis and with $\Gamma \cap \Delta$ the intersection of two basis, defined in the expected way.

Definition 1. Typed terms of $\mathcal{S} \ell \lambda$-calculus are defined by using a type assignment proving judgements of the shape $\Gamma \vdash \mathrm{M}: \sigma$, in Table 1

Note that only higher-order variables are subject to syntactical constraints. Except for the $\ell$ if construction typed by an additive rule doing an implicit contraction, higher-order variables are treated linearly. Ground and stable variables belong to distinct kinds only for sake of simplicity, their free use implies that $\mathcal{S} \ell \lambda$-calculus is not syntactically linear (in the sense of [10]).

Free variables of terms are defined as expected. A term $M$ is closed if and only if $F V(M)=\emptyset$, otherwise $\mathrm{M}$ is open. Terms are considered up to $\alpha$-equivalence, namely a bound variable can be renamed provided no free variable is captured. Moreover, $M[\underline{n} / \mathrm{y}], M[N / f]$ and $M[N / F]$ denote the expected capture-free substitutions.

Definition 2. We denote $\leadsto$ the firing (without any context-closure) of one of the following rules:

$$
\begin{aligned}
& \left(\lambda \mathrm{f}^{\sigma-\circ \tau} . \mathrm{M}\right) \mathrm{N} \sim_{\beta} \mathrm{M}[\mathrm{N} / \mathrm{f}] \quad\left(\lambda \mathrm{z}^{l} . \mathrm{M}\right) \underline{\mathrm{n}} \sim_{l} \mathrm{M}[\underline{\mathrm{n}} / \mathrm{z}] \quad \mu F . \mathrm{M} \sim_{\mathrm{Y}} \mathrm{M}[\mu F . \mathrm{M} / F] \\
& \operatorname{pred}(\operatorname{succ} \underline{\mathrm{n}}) \leadsto \underline{\sim} \underline{\mathrm{n}} \quad \ell \text { if } \underline{\mathbb{Q}} \underline{\mathrm{L} R} \sim_{\delta} \mathrm{L} \quad \text { if } \underline{\mathrm{n}+1} \mathrm{LR} \sim_{\delta} \mathrm{R}
\end{aligned}
$$

We call redex each term or sub-term having the shape of a left-hand side of rules defined above. We denote $\rightarrow$ se the contextual closure of $\leadsto$. Moreover, we denote $\rightarrow_{S \ell}^{*}$ and $=$ se respectively, the reflexive and transitive closure of $\rightarrow s$ and the reflexive, symmetric and transitive closure of $\rightarrow s e$.

We remark that $\sim_{\beta}$ formalises a call-by-name parameter passing in case of an higherorder argument. On the other hand, $\sim_{\iota}$ formalises a call-by-value parameter passing, namely the reduction can fire only when the argument is a numeral. As done in [2], it is easy to prove properties as subject-reduction, post-position of $\delta$-rules in a sequence of reductions, the confluence and a standardisation theorem. 


\section{Categorical model of $\mathcal{S} \ell \lambda$-calculus}

In this section we define the categorical model of $\mathcal{S} \ell \lambda$-calculus and we prove its soundness with respect to $=s \ell$. We assume some familiarity with the notions of monoidal categories, comonoids, comonads, adjunctions and monoidal functors. For an introduction, see [7]. We begin by recalling the definition of Linear Category, given by Benton, Bierman, Hyland and de Paiva, which proposes a categorical notion of model for Intuitionistic Linear Logic.

Definition 3 (Linear Category [1]). A Linear Category $\mathcal{L}=\langle\mathbb{L}, !, \delta, \varepsilon, q, d, e\rangle$ consists of (1) a symmetric monoidal closed category $\langle\mathbb{L}, \otimes,-0, \mathbf{1}\rangle ;$; (2) a symmetric monoidal comonad called exponential comonad $\left\langle!, \delta, \varepsilon, q_{A, B}, q_{1}\right\rangle: \mathbb{L} \rightarrow \mathbb{L}$, such that (i) for every free !-coalgebra $\left\langle! A, \delta_{A}\right\rangle$ there are two distinguished monoidal natural transformations with components $d_{A}: ! A \rightarrow ! A \otimes ! A$ and $e_{A}: ! A \rightarrow \mathbf{1}$ which form a commutative comonoid and are coalgebra morphisms; (ii) whenever $f:\left\langle! A, \delta_{A}\right\rangle \rightarrow\left\langle! B, \delta_{B}\right\rangle$ is a coalgebra morphism between free coalgebras, then it is also a comonoid morphism.

A Linear Category provides a sound categorical model of Intuitionistic Linear Logic [1]. A $\mathcal{S} \ell \lambda$-category will be a Linear Category, thus every $\mathcal{S} \ell \lambda$-Category is a model of Intuitionistic Linear Logic. However, it is necessary to augment it with other opportune features, in order to generate a sound categorical model of $\mathcal{S} \ell \lambda$-calculus.

\subsection{Numerals}

First of all, we need a canonical object to interpret ground type $\iota$ and opportune morphisms to interpret successor and predecessor. The following definition is an adaptation to monoidal categories of the definition of "simple object of numerals" given in [4].

Definition 4 (Monoidal Object of Numerals). Let $\mathbb{C}$ be a symmetric monoidal category. Let $\mathrm{N}$ be an object equipped with two morphisms $0: \mathbf{1} \rightarrow N$ and succ $: N \rightarrow N$. A numeral $n: \mathbf{1} \rightarrow N$ is defined inductively as the map $0: \mathbf{1} \rightarrow N$ for the base case, while the map $n+1: \mathbf{1} \rightarrow N$ is equal to succ $\circ n . N$ is said to be a monoidal object of numerals when it is also equipped with a morphism pred $: N \rightarrow N$ such that the following diagram commutes

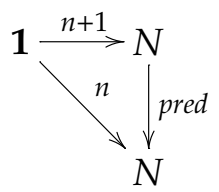

The definition above is very weak. It is in fact not required that given two numerals $m: \mathbf{1} \rightarrow N$ and $n: \mathbf{1} \rightarrow N$ with $n \neq m$ (viewed as numbers), they are distinct morphisms in $\mathbb{C}$. Moreover the definition given above does not allow to represent neither recursive nor primitive recursive functions in $\mathbb{C}$. An analogous situation is also present in in the definition of simple object of numerals given in [4].

For sake of completeness, we now compare the above definition with the definition given in [11]. It extends the notion of natural number object, which was specifically defined for cartesian categories in [14], to any monoidal category.

Definition 5 ([11, 6]). Let $\mathbb{C}$ be a symmetric monoidal closed category. By a natural number object in $\mathbb{C}$ we mean an object $N$ and two morphisms $0: \mathbf{1} \rightarrow N$ and succ $: N \rightarrow N$ such that, given any pair 
of morphisms $c: \mathbf{1} \rightarrow A$ and $f: A \rightarrow A$ there is a unique $h: N \rightarrow A$ making the following diagrams commute.
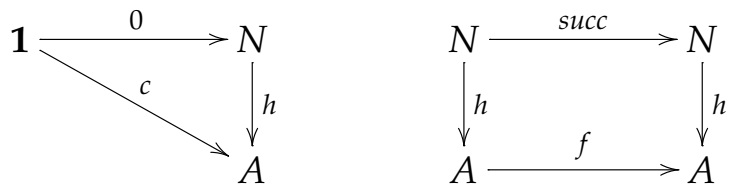

In [11], Paré and Román show that in any symmetric monoidal category $\mathbb{C}$ with a natural number object, the theory of primitive recursive functions can be developed. This is done by considering the category of commutative co-monoids in $\mathbb{C}$, which is cartesian [11] and where the theory of natural number objects is well developed. In detail, if $\left\langle C, d_{C}, e_{C}\right\rangle$ and $\left\langle D, d_{D}, e_{D}\right\rangle$ are two commutative co-monoids, then its cartesian product is given by $\left\langle C \otimes D, d_{C} \otimes d_{D}, e_{C} \otimes e_{D}\right\rangle$, while the pairing and the projections are defined as

$$
\begin{array}{rll}
\pi_{1} & \text { is the composite of } & C \otimes D \stackrel{i d_{C} \otimes e_{D}}{\rightarrow} C \otimes \mathbf{1} \stackrel{\varrho}{\rightarrow} C \\
\pi_{2} & \text { is the composite of } & C \otimes D \stackrel{e_{C} \otimes i d_{D}}{\rightarrow} \mathbf{1} \otimes D \stackrel{\lambda}{\rightarrow} D \\
\langle f, g\rangle & \text { is the composite of } & E \stackrel{d_{E}}{\rightarrow} E \otimes E \stackrel{f \otimes g}{\rightarrow} C \otimes D
\end{array}
$$

for $f: E \rightarrow C$ and $g: E \rightarrow D$. The terminal object is $\mathbf{1}$.

More specifically in [11] it is shown that if $N$ is a natural number object, then it is a commutative co-monoid, by taking the morphisms $w_{N}: N \rightarrow \mathbf{1}$ and $c_{N}: N \rightarrow N \otimes N$ to be the unique morphisms making the following diagrams commute
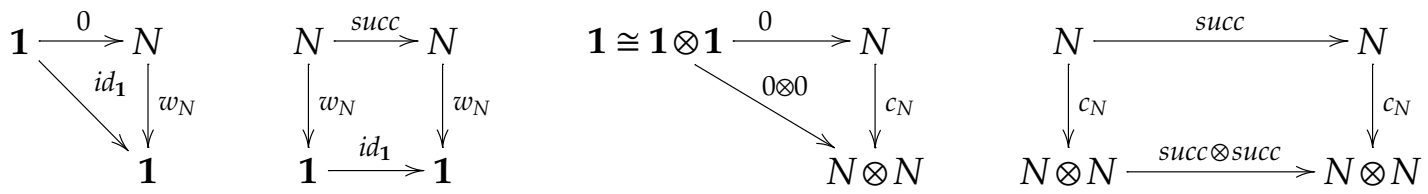

Furthermore $0: \mathbf{1} \rightarrow N$ and succ $: N \rightarrow N$ are both comonoid morphisms. Thus, all numerals are co-monoid morphisms, and all primitive recursive functions can be represented, in the same way as they were represented in a Cartesian Category [14]. Observe again that the above definition of natural number object does not require that given two numerals $n: \mathbf{1} \rightarrow N$ and $m: \mathbf{1} \rightarrow N$ with $n \neq m$ (viewed as numbers) are distinct morphisms in $\mathbb{C}$. But in [11], it has been shown that if this holds and if $\mathbb{C}$ is monoidal closed, then $\mathbb{C}$ is equivalent to the one-object one-morphism category (an analogous fact holds also for Cartesian Closed Categories [4]).

The following proposition is a corollary of the above statement.

Proposition 1. Let $\mathbb{C}$ be a symmetric monoidal closed category with a natural number object $N$. Then $N$ is a monoidal object of numerals.

Proof. Let $h: N \rightarrow N \otimes N$ be the unique morphism making the following diagrams commute (the pairing and projections in the category of commutative comonoid of $\mathbb{C}$ are defined above).
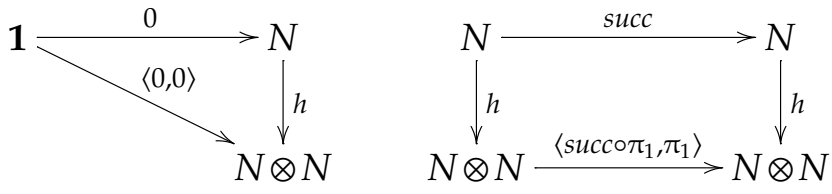
Thus, a choice for pred $: N \rightarrow N$ could be the following

$$
\text { pred is the composite of } N \stackrel{h}{\rightarrow} N \otimes N \stackrel{\pi_{2}}{\rightarrow} N
$$

It is not difficult to see that this choice of pred satisfies usual equation.

We now give a notion of natural number object in Linear Categories. We observe that a monoidal object of numerals is too weak, in order to be a sound interpretation of the type $\iota$ of S\&PCF. The structure of monoidal object of numerals should be enriched to obtain an exponential object of numerals; it will be a monoidal object of numerals with additional morphisms allowing to duplicate and weaken occurrences of them and whose other morphisms respects the comonoidal structure induced by the exponential co-monad.

Definition 6 (Exponential object of numerals). Let $\langle\mathbb{L}, !, \delta, \varepsilon, q, d, e\rangle$ be a Linear Category. An exponential object of numerals is a!-coalgebra $\langle N, p\rangle$ such that

1. $N$ is a monoidal object of numerals.

2. There exists two morphisms $w_{N}: N \rightarrow \mathbf{1}$ and $c_{N}: N \rightarrow N \otimes N$ which form a commutative co-monoid and are such that

(a) $0: \mathbf{1} \rightarrow N$ and succ $: N \rightarrow N$ are both co-algebras and co-monoid morphisms.

(b) $p: N \rightarrow ! N$ is a co-monoid morphism.

Proposition 2. Let $\langle\mathbb{L}, !, \delta, \varepsilon, q, d, e\rangle$ be a Linear Category and let $N$ be a natural number object such that $\langle N, p\rangle$ is a !-coalgebra satisfying

1. $p: N \rightarrow ! N$ is a co-monoid morphism.

2. $0: \mathbf{1} \rightarrow N$ and succ $: N \rightarrow N$ are coalgebra morphisms

Then $\langle N, p\rangle$ is an exponential object of numerals.

\section{$3.2 \mathcal{S} \ell \lambda$-category}

We now introduce our categorical model, by defining the notion of $\mathcal{S} \ell \lambda$-category whose morphisms will denote $\mathcal{S} \ell \lambda$-terms. We introduce some notation on the symmetric monoidal closed category $\mathbb{L}$ first. We let $\gamma_{A, B}: A \otimes B \cong B \otimes A$ the tensorial symmetric law. We denote with curry( $(-): \mathbb{L}(C \otimes A, B) \rightarrow \mathbb{L}(C, A \multimap B)$ the isomorphism induced by the canonical adjunction. When $C=A \multimap B$, we denote with eval $: A \multimap B \otimes A \rightarrow B$ the (unique!) morphism such that curry $($ eval $)=i d_{A \multimap B}$.

An $\mathcal{S} \ell \lambda$-category is a Linear Category admitting an exponential object of numerals, together with a "conditional-like" morphism and a fix-point morphism for every object $B$ in the Kleisli category over the co-monad !, which is cartesian closed. This leads to the following definition. Definition 7 ( $\mathcal{S} \ell \lambda$ Category). A $\mathcal{S} \ell \lambda$ Category is a linear category $\mathcal{L}=\langle\mathbb{L}, !, \delta, \varepsilon, q, d, e\rangle$ such that Numerals. $\mathbb{L}$ admits and exponential object of numerals $\langle N, p\rangle$.

Conditional Operator. $\mathbb{L}$ is cartesian and there exists a morphism lif $: N \otimes(N \times N) \rightarrow N$ such that, for all $f, g: \mathbf{1} \rightarrow N$, the following diagram commutes

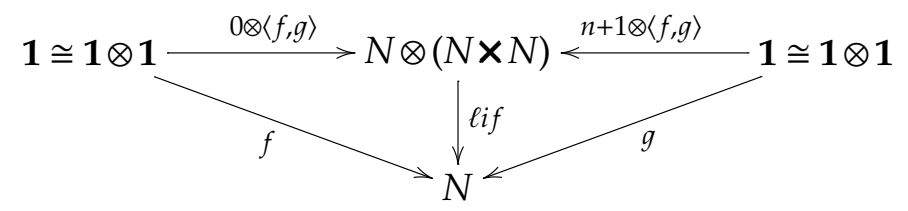


Fix-Point Operator. The Kleisli category $\mathbb{L}_{!}$(which is Cartesian Closed) admits a fix-point operator $f_{i x}: !(! B \multimap B) \rightarrow B$ for any object $B$. We remind that, by the Kleisli-construction, we have that the following diagram commutes.

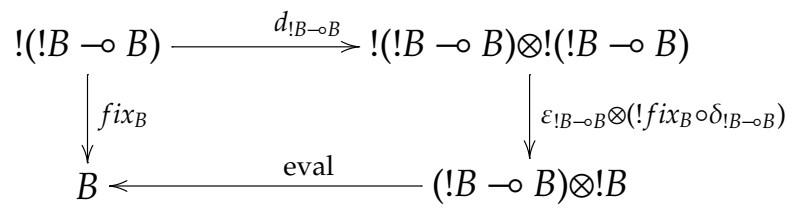

Definition 8 (Categorical $\mathcal{S} \ell \lambda$-model). A categorical $\mathcal{S} \ell \lambda$-model consists of

- A $\mathcal{S} \ell \lambda$ Category $\left\langle\mathcal{L}, N, p, c_{N}, w_{N}, \ell i f\right.$, fix $\rangle$, where $\mathcal{L}=\langle\mathbb{L}, !, \delta, \varepsilon, q, e, d\rangle$.

- A mapping associating to every Se $\mathcal{S}$-type $\sigma$, an object $\llbracket \sigma \rrbracket$ of $\mathbb{L}$ such that $\llbracket \iota \rrbracket=N$ and $\llbracket \sigma \multimap \tau \rrbracket=$ $\llbracket \sigma \rrbracket \multimap \llbracket \tau \rrbracket$.

- Given a basis $\Gamma$ we define $\llbracket \Gamma \rrbracket$ by induction as $\llbracket \emptyset \rrbracket=\mathbf{1}, \llbracket \mathrm{x}^{\sigma}, \Delta \rrbracket=\llbracket \sigma \rrbracket \otimes \llbracket \Delta \rrbracket$ and $\llbracket F^{\sigma}, \Delta \rrbracket=$ $! \llbracket \sigma \rrbracket \otimes \llbracket \Delta \rrbracket$. Moreover, given a basis $\Gamma$ such that $\Gamma^{\iota}=\mathrm{x}_{1}^{\iota}, \ldots, \mathrm{x}_{\mathrm{n}}^{\iota}\left(\right.$ resp. $\left.\Gamma^{*}=F_{1}^{\sigma_{1}}, \ldots, F_{n}^{\sigma_{n}}\right)$ we denote with $p_{\Gamma}=p \otimes \cdots \otimes p$-times (resp. $\delta_{\Gamma}=\delta_{\llbracket \sigma_{1} \rrbracket} \otimes \cdots \otimes \delta_{\llbracket \sigma_{n} \rrbracket}$ ).

Given a term $\mathrm{M}$ such that $\Gamma \vdash \mathrm{M}: \sigma$ we associate it a morphism $\llbracket \Gamma \vdash \mathrm{M}: \sigma \rrbracket: \llbracket \Gamma \rrbracket \rightarrow \llbracket \sigma \rrbracket$, such that

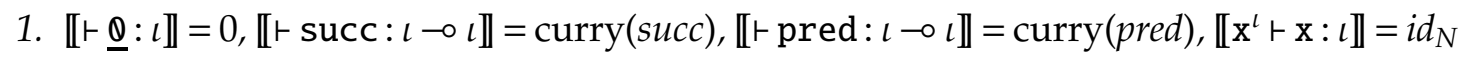

2. $\llbracket \mathrm{f}^{\bar{\sigma}-\tau \tau} \vdash \mathrm{f}: \sigma \multimap \tau \rrbracket^{\mathbb{L}}=i d_{\llbracket \sigma-\sigma \tau \rrbracket}, \llbracket F^{\sigma} \vdash F: \sigma \rrbracket=\varepsilon_{\llbracket \sigma \rrbracket}$

3. $\llbracket \Gamma^{\iota}, \Delta^{*} \vdash \mu F . M: \sigma \rrbracket=f i x_{\llbracket \sigma \rrbracket} \circ ! \operatorname{curry}\left(\llbracket \Gamma^{\iota}, \Delta^{*}, F^{\sigma} \vdash M: \sigma \rrbracket\right) \circ q \circ\left(p_{\Gamma} \otimes \delta_{\Delta}\right)$

4. $\llbracket \Gamma \vdash \lambda \mathbf{x}^{\sigma} . M: \sigma \multimap \tau \rrbracket=\operatorname{curry}\left(\llbracket \Gamma, \mathrm{x}^{\sigma} \vdash \mathrm{M}: \tau \rrbracket\right)$

5. $\llbracket \Gamma, \varkappa_{1}^{\sigma_{1}}, \boldsymbol{u}_{2}^{\sigma_{2}}, \Delta \vdash \mathbb{M}: \tau \rrbracket=\llbracket \Gamma, \varkappa_{2}^{\sigma_{2}}, \varkappa_{1}^{\sigma_{1}}, \Delta \vdash \mathbb{M}: \tau \rrbracket \circ\left(i d_{\llbracket \Gamma \rrbracket^{\mathbb{L}}} \otimes \gamma_{\llbracket \sigma_{1} \rrbracket, \llbracket \sigma_{2} \rrbracket} \otimes i d_{\llbracket \Delta \rrbracket}\right)$

6. $\llbracket \Gamma, \Delta \vdash \mathrm{MN}: \tau \rrbracket=\operatorname{eval} \circ(\llbracket \Gamma \vdash \mathrm{M}: \sigma \multimap \tau \rrbracket \otimes \llbracket \Delta \vdash \mathrm{N}: \sigma \rrbracket)$.

7. $\llbracket \Gamma, \Delta \vdash \ell$ if M L R : $\iota \rrbracket=\ell$ if $\circ(\llbracket \Gamma \vdash M: \iota \rrbracket \otimes\langle\llbracket \Delta \vdash \mathrm{L}: \iota \rrbracket, \llbracket \Delta \vdash \mathrm{R}: \iota \rrbracket\rangle)$.

8. $\llbracket \Gamma, \mathrm{x}^{\iota} \vdash \mathrm{M}\left[\mathrm{x} / \mathrm{x}_{1}, \mathrm{x}_{2}\right]: \tau \rrbracket=\llbracket \Gamma, \mathrm{x}_{1}{ }^{l}, \mathrm{x}_{2}{ }^{\iota} \vdash \mathrm{M}: \tau \rrbracket \circ i d_{\llbracket \Gamma \rrbracket} \otimes c_{N}$

9. $\llbracket \Gamma, F^{\sigma} \vdash \mathrm{M}\left[F / F_{1}, F_{2}\right]: \tau \rrbracket=\llbracket \Gamma, F_{1}{ }^{\sigma}, F_{2}{ }^{\sigma} \vdash \mathrm{M}: \tau \rrbracket \circ i d_{\llbracket \Gamma \rrbracket} \otimes d_{\llbracket \sigma \rrbracket}$

10. $\llbracket \Gamma, \mathrm{x}^{\imath} \vdash \mathrm{M}: \tau \rrbracket=\llbracket \Gamma \vdash \mathrm{M}: \tau \rrbracket \circ i d_{\llbracket \Gamma \rrbracket} \otimes w_{N}$

11. $\llbracket \Gamma, F^{\sigma} \vdash \mathrm{M}: \tau \rrbracket=\llbracket \Gamma \vdash \mathrm{M}: \tau \rrbracket \circ i d_{\llbracket \Gamma \rrbracket} \otimes e_{\llbracket \sigma \rrbracket}$

\section{Soundness}

The following theorem shows that the three kinds of syntactical substitutions are modelled by categorical composition of morphisms. Let us observe that the substitution of a ground or higher-order variable respectively with a numeral or a term is modelled directly with the composition in $\mathbb{L}$, while the substitution of a stable variable with a term is modelled with the composition in the category of coalgebras.

Theorem 1 (Semantical Substitution Lemma).

1. Let $\mathrm{M}$ be such that $\Gamma, \mathrm{x}^{l}, \Delta \vdash \mathrm{M}: \sigma$. Then $\llbracket \Gamma, \Delta \vdash \mathrm{M}[\underline{\mathrm{n}} / \mathrm{x}]: \sigma \rrbracket=\llbracket \Gamma, \mathrm{x}^{\iota}, \Delta \vdash \mathrm{M}: \sigma \rrbracket \circ\left(i d_{\llbracket \Gamma \rrbracket} \otimes n \otimes i d_{\llbracket \Delta \rrbracket}\right)$.

2. Let $\mathrm{M}, \mathrm{N}$ be such that $\Gamma, \mathrm{f}^{\sigma} \vdash \mathrm{M}: \tau$ and $\Delta \vdash \mathrm{N}: \sigma$, with $\Gamma \cap \Delta=\emptyset$. Then $\llbracket \Gamma, \Delta \vdash \mathrm{M}[\mathrm{N} / \mathrm{f}]: \tau \rrbracket=\llbracket \Gamma, \mathrm{f}^{\sigma} \vdash \mathrm{M}: \tau \rrbracket \circ\left(i d_{\llbracket \Gamma \rrbracket} \otimes \llbracket \Delta \vdash \mathrm{N}: \sigma \rrbracket\right)$.

3. Let $\mathrm{M}, \mathrm{N}$ be such that $\Gamma, F^{\sigma} \vdash \mathrm{M}: \tau$ and $\Delta_{1}^{\iota}, \Delta_{2}^{*} \vdash \mathrm{N}: \sigma$, with $\Gamma \cap \Delta_{1} \cap \Delta_{2}=\emptyset$. Then $\llbracket \Gamma, \Delta_{1}^{\iota}, \Delta_{2}^{*} \vdash \mathrm{M}[\mathrm{N} / F]: \tau \rrbracket=\llbracket \Gamma, F^{\sigma} \vdash \mathrm{M}: \tau \rrbracket \circ\left(i d_{\llbracket \Gamma \rrbracket} \otimes\left(! \llbracket \Delta_{1}^{\iota}, \Delta_{2}^{*} \vdash \mathrm{N}: \sigma \rrbracket \circ q \circ\left(p_{\Delta_{1}} \otimes \delta_{\Delta_{2}}\right)\right)\right)$. 
Proof. All the proofs follow by induction on the derivation of the typing judgements. The key point is to show that the transformations induced by the typing rules are natural on the unchanged components of the sequent. More details can be found in [12].

Theorem 2 (Soundness). Let $\mathrm{M}, \mathrm{N}$ be such that $\Gamma \vdash \mathrm{M}: \sigma$ and $\Gamma \vdash \mathrm{N}: \sigma$. Then,

$$
\text { if } \mathrm{M}={ }_{\mathcal{S e}} \mathrm{N} \text { then } \llbracket \Gamma \vdash \mathrm{M}: \sigma \rrbracket=\llbracket \Gamma \vdash \mathrm{N}: \sigma \rrbracket
$$

Proof. The proof is by induction on the derivation of $\mathrm{M}={ }_{S \ell} \mathrm{N}$. We develop only the case $\mathrm{M}={ }_{S \ell} \mathrm{N}$ since $\mathrm{M} \sim \mathrm{Y}$. Thus $\mathrm{M}=\mu F \cdot \mathrm{M}_{1}$ and $\mathrm{N}=\mathrm{M}_{1}\left[\mu F . \mathrm{M}_{1} / F\right]$. To help with the notation, in the proofs we relax a bit the definition of basis and we add types prefixed with a ! to the syntax of types. Thus, given a basis $\Gamma=\varkappa_{1}^{\sigma_{1}}, \ldots, \varkappa_{n}^{\sigma_{n}}$, we denote with $! \Gamma=\varkappa_{1}^{! \sigma_{1}}, \ldots \mathcal{\varkappa}_{n}^{! \sigma_{n}}$ (where ! is just a syntactical annotation which will be interpreted with the corresponding categorical operator) and we adapt in the canonical way the interpretation function on the so obtained types and basis. First of all, if we let $f=\llbracket \Gamma^{\iota}, \Delta^{*}, F^{\sigma} \vdash \mathrm{M}_{1}: \sigma \rrbracket$, let us observe that the following diagram commutes

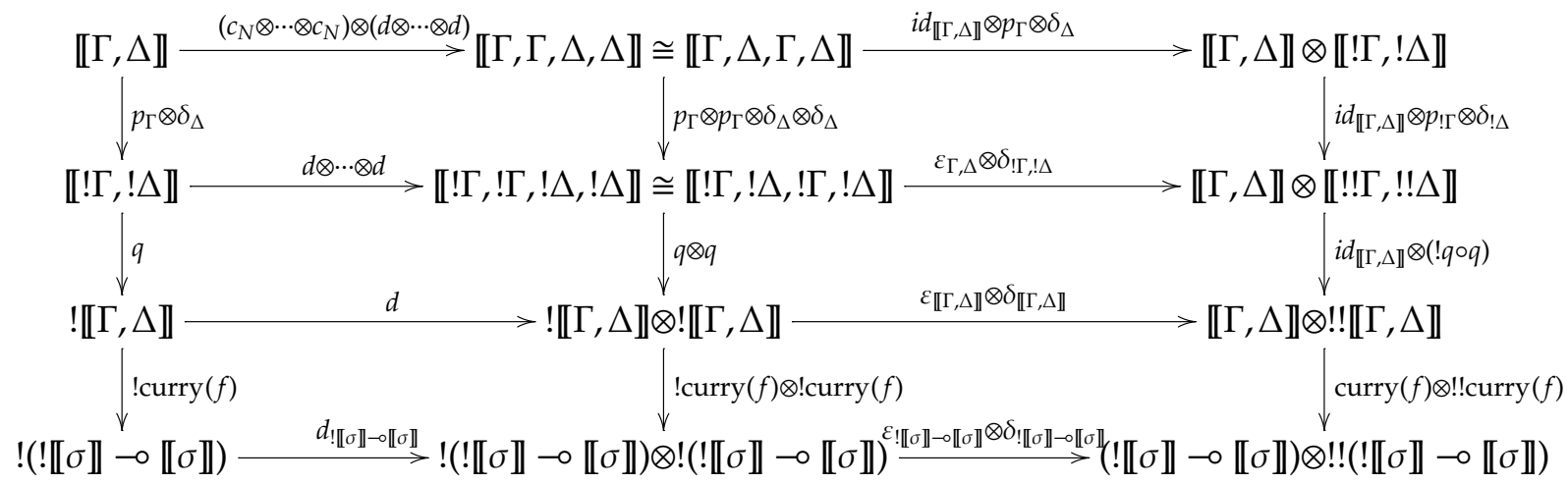

where the left square on the top commutes since $p$ and $\delta$ are co-monoid morphisms, the right square on the top commutes since $p$ and $\delta$ are co-algebras (observe that we used both commutative diagrams of the definition of co-algebra) and by bi-functoriality, the left square on the middle commutes since $d$ is a monoidal natural transformation, the right square on the middle commutes since $\delta$ and $\varepsilon$ are monoidal natural transformations, and finally the two squares on the bottom commutes respectively because being !curry $(f)$ a co-algebra morphism between free co-algebra, it is also a co-monoid morphism, by naturality of $\varepsilon$ and $\delta$ and by bi-functoriality. Thus, we have,

$$
\begin{aligned}
& \llbracket \Gamma^{\iota}, \Delta^{*} \vdash \mathrm{M}: \sigma \rrbracket=f i x_{\llbracket \sigma \rrbracket^{\circ}} ! \operatorname{curry}(f) \circ q \circ\left(p_{\Gamma} \otimes \delta_{\Delta}\right) \\
& =\operatorname{eval} \circ\left(\varepsilon_{! \llbracket \sigma \rrbracket-\curvearrowleft \llbracket \sigma \rrbracket} \otimes\left(! f i x_{\llbracket \sigma \rrbracket} \circ \delta_{! \llbracket \sigma \rrbracket-\curvearrowleft \llbracket \sigma \rrbracket}\right)\right) \circ d_{! \llbracket \sigma \rrbracket-\llbracket \sigma \rrbracket} \circ ! \text { curry }(f) \circ q \circ\left(p_{\Gamma} \otimes \delta_{\Delta}\right) \\
& =\operatorname{eval} \circ\left(\operatorname{curry}(f) \otimes\left(! f i x_{\llbracket \sigma \rrbracket} \circ ! ! \operatorname{curry}(f) \circ(! q \circ q) \circ\left(p_{! \Gamma} \otimes \delta_{! \Delta}\right)\right) \circ\left(p_{\Gamma} \otimes \delta_{\Delta}\right)\right) \circ\left(\left(c_{N} \otimes \cdots \otimes c_{N}\right) \otimes(d \otimes \cdots \otimes d)\right) \\
& =f \circ i d_{\llbracket \Gamma, \Delta \rrbracket} \otimes\left(! \llbracket \Gamma^{\iota}, \Delta^{*} \vdash \mu F . M: \sigma \rrbracket \circ q \circ\left(p_{\Gamma} \otimes \delta_{\Delta}\right)\right) \circ\left(\left(c_{N} \otimes \cdots \otimes c_{N}\right) \otimes(d \otimes \cdots \otimes d)\right) \\
& =\llbracket \Gamma^{l}, \Delta^{*}, \Gamma^{l}, \Delta^{*} \vdash \mathrm{N}: \sigma \rrbracket \circ\left(\left(c_{N} \otimes \cdots \otimes c_{N}\right) \otimes(d \otimes \cdots \otimes d)\right)
\end{aligned}
$$

where in the second line we used the fix-point law, in the third line we use the commutativity of the above diagram, in the fourth line we use the definition of interpretation, the naturality of $q$ and the fact that the category is monoidal closed. Finally, in the fifth line we use Theorem 1 point (3). Then we can conclude by definition of interpretation. 


\section{Instances of $\mathcal{S} \ell \lambda$-categories}

In this section, we show three interesting concrete instances of $\mathcal{S} \ell \lambda$-category, in the setting of Scott Domains and Coherence Spaces. By means of the results proved in the previous section this three instances gives models in which the $\mathcal{S} \ell \lambda$-calculus can be soundly interpreted.

\subsection{Scott Domains and strict continuous functions}

Let StrictBcdom (Strict Bounded Complete Domains) be the category obtained by taking as objects $\omega$-algebraic bounded complete partial orders (or Scott domains) and as morphisms strict continuous functions, namely those continuous functions that map the bottom element of the source object to the bottom element of the target. This category is monoidal closed, by taking the tensor product $A \otimes B$ to be the smash product $A \wedge B=\{\langle a, b\rangle \mid a \in A \backslash\{\perp\}, b \in B \backslash\{\perp\}\} \cup\{\perp\}$, the unit of the tensor product 1 to be the Sierpinsky Domain $\{\perp, T\}$ with $\perp \leq T$ and the function space $A \multimap B$ consisting of all strict maps between $A$ and $B$ under the point-wise order. Moreover if we take as exponential comonad!, the lifting constructor $(-)_{\perp}$, we obtain a linear category; we remind that, given a Scott Domain $A$, the domain $A_{\perp}$ is obtained from $A$ by adding a new least element below the bottom of $A$ (for more details see [13]). Observe that the Kleisli category over the comonad $(-)_{\perp}$ is the usual category of Scott Domains and continuous functions.

We can prove that StrictBcdom is a $\mathcal{S} \ell \lambda$ Category, by taking $N$ to be the usual flat domain of natural numbers with the coalgebra $p: N \rightarrow N_{\perp}$ such that $p(n)=n$ for all $n \neq \perp$. $N$ is a commutative comonoid, by taking $w_{N}: N \rightarrow \mathbf{1}$ be such that $w_{N}(n)=\top$ and $c_{N}: N \rightarrow N \otimes N$ be such that $c_{N}(n)=\langle n, n\rangle$ for all $n \neq \perp$. StrictBcdom is cartesian, by taking $A \times B$ to be the usual cartesian product of Scott Domains. Thus we can define $\ell$ if $: N \otimes(N \times N) \rightarrow N$ to be such that $\ell$ if $(c)=m_{1}$ if $c=\left\langle 0,\left\langle m_{1}, m_{2}\right\rangle\right\rangle, \ell$ if $(c)=m_{2}$ if $c=\left\langle n,\left\langle m_{1}, m_{2}\right\rangle\right\rangle$ and $n \neq 0$ and $\ell$ if $(c)=\perp$ otherwise. Finally, it follows easily by Knaster-Tarsky's Fix Point Theorem that the considered category admits fix point for every object. This model is shown to be adequate with respect to the operational semantics of $\mathcal{S} \ell \mathrm{PCF}$ in [12].

\subsection{Coherence Spaces.}

A coherence space is a pair $X=\left\langle|X|, \bigcirc_{X}\right\rangle$, consisting of a finite or countable set of tokens $|X|$ called web and a binary reflexive symmetric relation on $|X|$ called coherence relation. The set of cliques of $X$ is given by $C l(X)=\left\{x \subseteq|X| \mid a, b \in x \Rightarrow a \triangle_{X} b\right\}$. This set ordered by inclusion forms a Scott Domain whose set of finite elements is the set $C l_{\text {fin }}(X)$ of finite cliques. Two cliques $x, y \in C l(X)$ are compatible when $x \cup y \in C l(X)$. A continuous function $f: C l(X) \rightarrow C l(Y)$ is stable when it preserves intersections of compatible cliques. A stable function $f: \mathrm{Cl}(X) \rightarrow \mathrm{Cl}(Y)$ is linear when it is strict and preserves unions of compatibles cliques. Given a linear function $f: C l(X) \rightarrow C l(Y)$, we denote its trace with $\operatorname{tr}(f)=\{(a, b) \mid b \in f(\{a\})\}$. We say that a linear function $f$ is less or equal than $g$ according to the stable order when $\operatorname{tr}(f) \subseteq \operatorname{tr}(g)$.

Let Coh be the category of coherence spaces as objects and linear functions as morphisms. Given a coherence space $X$, we define $! X$ to be the coherence space having as web the set $C l_{f i n}(X)$ and as coherence relation, the compatibility relation between cliques. It is possible to prove that ! is an exponential comonad, thus Coh is a Linear Category [8]. Observe that the Kleisli category over the comonad ! is the category of Coherence Spaces and Stable Functions 
The model of SePCF we define in [10] is based on this category, and can be obtained as follows. As in previous section, we take $N$ to be the infinite flat domain of natural numbers, and we define $w_{N}, c_{N}$ in an analogous way as before, as well as $\ell$ if and the fix point operator. The !-coalgebra $p: N \rightarrow ! N$ is such that $\operatorname{tr}(p)=\{(n,\{n\}) \mid n \in \mathbb{N}\} \cup\{(n,\{\emptyset\}) \mid n \in \mathbb{N}\}$.

\subsection{Scott Domains and Linear Functions}

A similar construction as the one presented above can be obtained also in the Scott Domain setting. Let LinBcdom (Linear Bounded Complete Domains) be the category defined as follows. The objects are again Scott Domains. If $D$ is a Scott Domain, we write $D_{0}$ for its poset of finite elements; $D$ is obtained from $D_{0}$ by adding all suprema of directed subsets of $D_{0}$. The morphisms are linear maps, i.e. functions which preserves all existing suprema ( $f: D_{1} \rightarrow D_{2}$ is linear if for all bounded $X \subseteq D_{1}$ we have $f(\bigsqcup X)=\bigsqcup f(X)$, reminding that $\left.\bigsqcup \emptyset=\perp\right)$. The tensor product $D_{1} \otimes D_{2}$ classifies maps $D_{1} \times D_{2} \rightarrow D$ linear in each argument, while the unit of the tensor product 1 is the Sierpinsky Domain $\{\perp, T\}$ with $\perp \leq T$; the linear function space $B \multimap C$ consists of all linear functions from $B$ to $C$ ordered pointwise. The cartesian product is the usual cartesian product between Scott Domain. The exponential comonad can be described in terms of finite element. Given $D$, we let the set $(! D)_{0}$ to be the set obtained from $D_{0}$ by freely adding suprema of bounded finite subsets of $D_{0}$. We complete $(! D)_{0}$ with all directed limits, to obtain $! D$. The Kleisli category over the comonad is the usual category of Scott domains and continuous maps.

We can prove that LinBcdom is an $\mathcal{S} \ell \lambda$ category by taking $N$ to be the usual flat domain of natural numbers and defining $p: N \rightarrow ! N$ as $p(\perp)=\perp$ and $p(n)=\sqcup\{\perp, n\}, w_{N}: N \rightarrow \mathbf{1}$ as $w_{N}(\perp)=\perp$ and $w_{N}(n)=T, c_{N}: N \rightarrow N \otimes N$ as $c_{N}(\perp)=\perp$ and $c_{N}(n)=\langle n, n\rangle$.

\section{Towards Completeness}

In the previous sections we have proved the soundness of our interpretation and we have shown some concrete examples. Now, a natural question is whether the $\mathcal{S} \ell \lambda$-calculus is also complete with respect to the notion of $\mathcal{S} \ell \lambda$-category we have introduced so far or not.

The answer is negative. Indeed, the completeness of $\mathcal{S} \ell \lambda$-calculus with respect to the notion of $\mathcal{S} \ell \lambda$-model fails. To understand why, let us consider the judgment

$$
\Gamma, \Delta \vdash\left(\lambda \mathrm{x}^{l} . \mathrm{M}\right) \mathrm{N}: \tau
$$

where $\Gamma \vdash \lambda \mathrm{x} . \mathrm{M}: \iota \multimap \tau$ and $\Delta \vdash \mathrm{N}: \iota$. The interpretation of this judgment is

$$
\llbracket \Gamma, \Delta \vdash\left(\lambda \mathrm{x}^{\iota} . \mathrm{M}\right) \mathrm{N}: \tau \rrbracket=\mathrm{eval} \circ\left(\llbracket \Gamma \vdash \lambda \mathrm{x}^{\iota} . \mathrm{M}: \iota \multimap \tau \rrbracket \otimes \llbracket \Delta \vdash \mathrm{N}: \sigma \rrbracket\right)
$$

The term eval above represents the standard evaluation morphism of the symmetric monoidal closed category. So, in particular it is easy to verify that the above interpretation is equal to

$$
\llbracket \Gamma, \Delta \vdash \mathrm{M}\left[\mathrm{N} / \mathrm{x}^{l}\right]: \tau \rrbracket
$$

So, we clearly have:

$$
\llbracket \Gamma, \Delta \vdash\left(\lambda \mathrm{x}^{\iota} . \mathrm{M}\right) \mathrm{N}: \tau \rrbracket=\llbracket \Gamma, \Delta \vdash \mathrm{M}\left[\mathrm{N} / \mathrm{x}^{\iota}\right]: \tau \rrbracket
$$

Unfortunately, in the $\mathcal{S} \ell \lambda$-calculus we have

$$
\left(\lambda \mathrm{x}^{l} \cdot \mathrm{M}\right) \mathrm{N} \neq{ }_{\mathcal{S} \ell} \mathrm{M}\left[\mathrm{N} / \mathrm{x}^{\iota}\right]
$$




$$
\frac{\Gamma \vdash \mathrm{M}: \iota}{\Gamma \vdash \operatorname{promote}^{\iota}(\mathrm{M}): ! \iota}\left(\mathrm{pr}_{\iota}\right) \quad \frac{\Gamma \vdash \mathrm{M}: \iota \Delta \vdash \mathrm{N}: \sigma}{\Gamma, \Delta \vdash \operatorname{discard} \mathrm{M} \text { in } \mathrm{N}: \sigma}\left(\mathrm{ds}_{\iota}\right) \frac{\Gamma \vdash \mathrm{M}: \iota \quad \Delta, \mathrm{x}_{1}{ }^{\iota}, \mathrm{x}_{2}{ }^{\iota} \vdash \mathrm{N}: \sigma}{\Gamma, \Delta \vdash \operatorname{copy}^{\iota} \mathrm{M} \text { as } \mathrm{x}_{1}, \mathrm{x}_{2} \text { in } \mathrm{N}: \sigma}\left(\mathrm{cp}_{\iota}\right)
$$

Table 2: Intuitionistic Linear typed calculus ILL

unless $\mathrm{N}$ is a numeral. So we have a counterexample to completeness.

In order to recover completeness, we can adopt different stategies. First of all, note that the terms in the equation 1 above turn to be equivalent if we consider observational equivalence instead of the equivalence induced by the reduction rules. So we could consider $\mathcal{S} \ell \lambda$-terms modulo observational equivalence. For such a system the completeness should hold. Unfortunately, this corresponds to study the notion of model of the programming language built over the calculus instead of studying a model of the calculus itself. In our setting, this corresponds to study the notion of models for $\mathcal{S} \ell$ PCF instead of the $\mathcal{S} \ell \lambda$-calculus but this is not our aim here. A different perspective is to extend the term assignment system for ILL introduced in [1] by means of operators for numerals, conditional and fix points. This can be done by extending the typed calculus by the rules in Figure 2 and by rules for conditional and fix points. The reduction rules of the obtained calculus should be designed starting from the categorial equalities. So, for example we obtain rules as

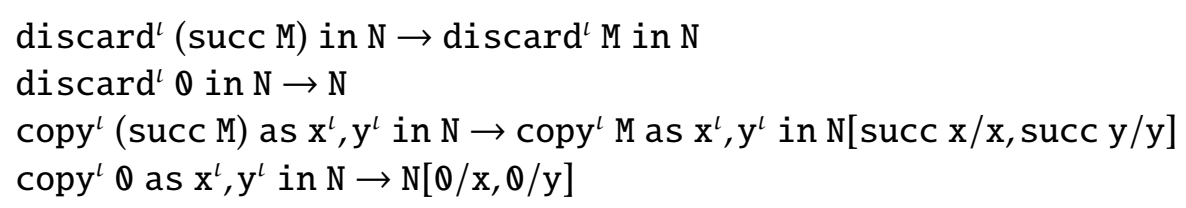

corresponding to the categorical equations deriving from the fact that zero and successor are comonoidal, as:

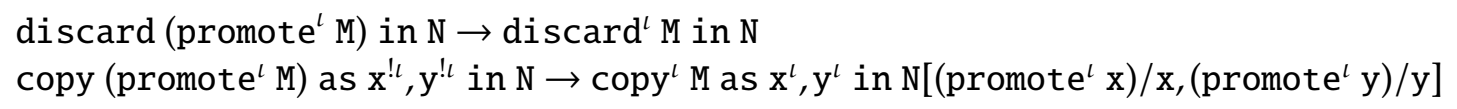

corresponding to the categorical equations deriving from the fact that promotion on numerals is comonoidal and as

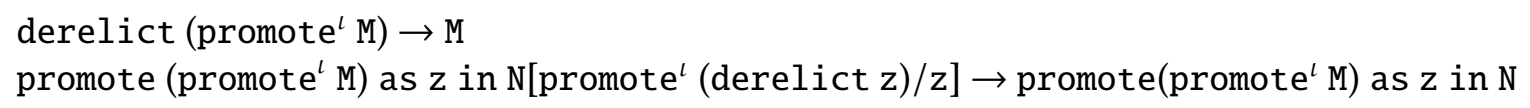

corresponding to the categorical equations deriving from the fact that promotion on numerals is also a !-coalgebra.

Finally, another interesting possibility is to change the standard interpretation function. In particular, we could change the interpretation of $\lambda$-abstractions binding ground variables by adapting standard technics already studied for the call by value $\lambda$-calculus.

\section{Conclusion}

In this work we have introduced the notion of $\mathcal{S} \ell \lambda$-category. Such a notion provide a categorical model for $\mathcal{S} \ell \lambda$-calculus introduced in [10]. We have shown that $\mathcal{S} \ell \lambda$-categories are sound for the interpretation of $\mathcal{S} \ell \lambda$-terms. Moreover, we have shown three concrete model examples in 
the setting of Scott Domains and Coherence Spaces.

The $\mathcal{S} \ell \lambda$-calculus is not complete with respect to $\mathcal{S} \ell \lambda$-category. In Section 6 we have sketched some approach in order to recover completeness. We plan to explore these approaches in future developments.

The concrete denotational models presented in Section 5 can be useful in the study of linear higher type computability [5, 9]. In this setting one interesting research theme is the study of paradigmatic programming languages fitting models founded on different higher type functionals.

On this matter, we have already obtained some preliminary results. In [10] the interpretation of S\&PCF into the category Coh is studied and a partial full abstraction result is presented. In future works we plan to systematically extend S SPCF with suitable operators in order to establish definability results with respect to Coh, StrictBcdom and LinBcdom.

\section{References}

[1] Nick Benton, G. M. Bierman, J. Martin E. Hyland \& Valeria de Paiva (1992): Linear $\lambda$-Calculus and Categorical Models Revisited. In: E. Börger, editor: Proceedings of the Sixth Workshop on Computer Science Logic - CSL, Lecture Notes in Computer Science 702. Springer-Verlag, pp. 61-84.

[2] Gérard Berry, Pierre-Louis Curien \& Jean-Jacques Lévy (1985): Full Abstraction for Sequential Languages: the State of the Art. In: M. Nivat \& John Reynolds, editors: Algebraic Semantics. Cambridge University Press, pp. 89-132.

[3] Marco Gaboardi \& Luca Paolini (2007): Syntactical, Operational and Denotational Linearity. In: Workshop LOGIC. Dedicated to Jean-Yves Girard on his 60th birthday. Certosa di Pontignano, Siena.

[4] J. Martin E. Hyland \& Luke C.-H. Ong (2000): On full abstraction for PCF: I, II, and III. Information and Computation 163(2), pp. 285-408.

[5] John R. Longley (2002): The sequentially realizable functionals. Annals of Pure and Applied Logic 117, pp. 1-93.

[6] Ian Mackie, Leopoldo Román \& Samson Abramsky (1993): An internal language for autonomous categories. Applied Categorical Structures 1(3), pp. 311-343.

[7] Saunders MacLane (1998): Categories for the Working Mathematician. Graduate Texts in Mathematics. Springer - Verlag. Second Edition.

[8] Paul-André Melliès (2003): Categorical Models of Linear Logic Revisited. Prépublication de l'équipe PPS.

[9] Luca Paolini (2006): A Stable Programming Language. Information and Computation 204(3), pp. 339-375.

[10] Luca Paolini \& Mauro Piccolo (2008): Semantically linear programming languages. In: Sergio Antoy \& Elvira Albert, editors: Proceedings of the 10th International ACM SIGPLAN Conference on Principles and Practice of Declarative Programming, July 15-17, 2008, Valencia, Spain. ACM, pp. 97-107.

[11] Robert Paré \& Leopoldo Román (1988): Monoidal categories with natural numbers object. Studia Logica 48(3), pp. 361-376.

[12] Mauro Piccolo (2009): Linearity and Behind in Denotational Semantics. Ph.D. thesis, Dipartimento di Informatica, Universitá di Torino/Laboratoire PPS, Université de Paris VII. In preparation.

[13] Alberto Pravato, Simona Ronchi Della Rocca \& Luca Roversi (1999): The call by value $\lambda$-calculus: a semantic investigation. Mathematical Structures in Computer Science 9(5), pp. 617-650. 
[14] Leopoldo Román (1989): Cartesian categories with natural numbers object. Journal of Pure and Applied Algebra 58(3), pp. 267-278. 